\title{
Transcriptome-wide analysis of intracranial artery in patients with moyamoya disease showing upregulation of immune response, and downregulation of oxidative phosphorylation and DNA repair
}

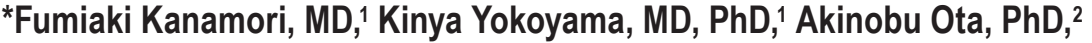 \\ Kazuhiro Yoshikawa, PhD, ${ }^{3}$ Sivasundaram Karnan, PhD, ${ }^{2}$ Mikio Maruwaka, MD, PhD, ${ }^{4}$ \\ Kenzo Shimizu, MD, ${ }^{5}$ Shinji Ota, MD, ${ }^{6}$ Kenji Uda, MD, PhD, ${ }^{1}$ Yoshio Araki, MD, PhD, ${ }^{1}$ \\ Sho Okamoto, MD, PhD, ${ }^{7}$ Satoshi Maesawa, MD, PhD, ${ }^{1}$ Toshihiko Wakabayashi, MD, PhD, ${ }^{1}$ and \\ Atsushi Natsume, MD, PhD'
}

\begin{abstract}
1Department of Neurosurgery, Nagoya University Graduate School of Medicine, Nagoya; ${ }^{2}$ Department of Biochemistry, Aich Medical University School of Medicine, Nagakute; ${ }^{3}$ Division of Research Creation and Biobank, Research Creation Support Center, Aichi Medical University, Nagakute; ${ }^{4}$ Department of Neurosurgery, Toyota Kosei Hospital, Toyota; ${ }^{5}$ Department of Neurosurgery, Kasugai Municipal Hospital, Kasugai; ${ }^{6}$ Department of Neurosurgery, Handa City Hospital, Handa; and ${ }^{7}$ Aichi Rehabilitation Hospital, Nishio, Japan
\end{abstract}

\begin{abstract}
OBJECTIVE Moyamoya disease (MMD) is a rare cerebrovascular disease characterized by progressive occlusion of the internal carotid artery and the secondary formation of collateral vessels. Patients with MMD have ischemic attacks or intracranial bleeding, but the disease pathophysiology remains unknown. In this study, the authors aimed to identify a gene expression profile specific to the intracranial artery in MMD.

METHODS This was a single-center, prospectively sampled, retrospective cohort study. Microsamples of the middle cerebral artery (MCA) were collected from patients with MMD $(n=11)$ and from control patients $(n=9)$. Using microarray techniques, transcriptome-wide analysis was performed.

RESULTS Comparison of MCA gene expression between patients with MMD and control patients detected 62 and 26 genes whose expression was significantly ( $p<0.001$ and fold change $>2$ ) up- or downregulated, respectively, in the MCA of MMD. Gene set enrichment analysis of genes expressed in the MCA of patients with MMD revealed positive correlations with genes involved in antigen processing and presentation, the dendritic cell pathway, cytokine pathway, and interleukin-12 pathway, and negative correlations with genes involved in oxidative phosphorylation and DNA repair. Microarray analysis was validated by quantitative polymerase chain reaction.
\end{abstract}

CONCLUSIONS Transcriptome-wide analysis showed upregulation of genes for immune responses and downregulation of genes for DNA repair and oxidative phosphorylation within the intracranial artery of patients with MMD. These findings may represent clues to the pathophysiology of MMD.

https://thejns.org/doi/abs/10.3171/2021.6.FOCUS20870

KEYWORDS moyamoya disease; transcriptome-wide analysis; intracranial artery

$\mathrm{M}$ OYAMOYA disease (MMD) is a rare cerebrovascular disease characterized by progressive stenosis of the internal carotid artery at its terminal portion and secondary formation of collateral vessels at the brain base; the latter are called moyamoya vessels. The stenosis of proximal large intracranial arteries decreases the cerebral blood flow, and the disease thus is associated with ischemic attacks. The resulting hemodynamic overstress in collateral vessels can induce pathological change, leading to intracranial hemorrhage. Surgical revascular-

ABBREVIATIONS cDNA = complementary DNA; DC = dendritic cell; GAPDH = glyceraldehyde 3-phosphate dehydrogenase; GSEA = gene set enrichment analysis; HIPK2 = homeodomain-interacting protein kinase 2; HUWE1 = HECT, UBA, and WWE domain containing E3 ubiquitin-protein ligase 1; IL-12 = interleukin-12; MCA = middle cerebral artery; $\mathrm{MMD}=$ moyamoya disease; $\mathrm{PCR}=$ polymerase chain reaction; $\mathrm{qPCR}=$ quantitative $\mathrm{PCR} ; \mathrm{RASAL} 3=\mathrm{RAS}$ protein activator-like $3 ; \mathrm{RHOQ}=$ ras homolog family member $\mathrm{Q} ; \mathrm{RNF213}$ = ring finger protein 213; STA = superficial temporal artery; TSPAN2 = tetraspanin 2.

SUBMITTED October 9, 2020. ACCEPTED June 18, 2021.

INCLUDE WHEN CITING DOI: 10.3171/2021.6.FOCUS20870.

${ }^{*}$ F. Kanamori and K. Yokoyama contributed equally to this work. 


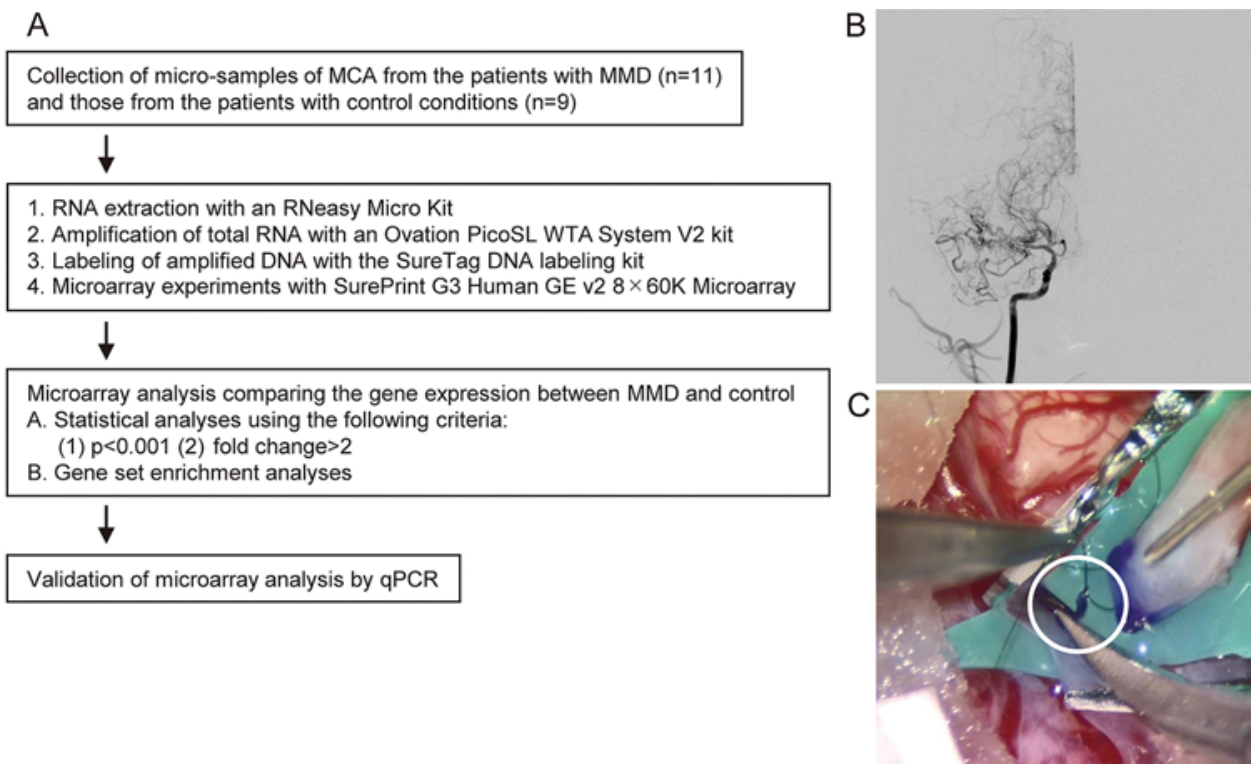

FIG. 1. A: Flow diagram of the study. B: Angiogram (anteroposterior view) obtained from a 43-year-old female with MMD. The preoperative right common carotid shows stenosis at the terminal portion of the internal carotid artery and moyamoya vessels. C: Intraoperative image of arteriotomy at the cortical segment of the MCA. A microsample was obtained from the tissue in the white circle, and this specimen was used for RNA extraction.

ization, including superficial temporal artery (STA)-tomiddle cerebral artery (MCA) anastomosis, is known as an effective method to reduce the risk of subsequent stroke and rebleeding. 1,2

Although the c.14576G $>$ A variant of ring finger protein 213 (RNF213) has been identified as a susceptibility variant for MMD in patients of East Asian background, ${ }^{3,4}$ little is known about the disease etiology and pathophysiology. The main histopathological characteristics of MMD are fibrous thickening of the intima and attenuation of the media in the terminal portion of the internal carotid artery; these histopathological characteristics are also observed in the cortical segment of the MCA. ${ }^{5-7}$ Due to the difficulty of extracting sufficient protein or RNA from the microsamples of MCA that can be obtained during surgery, few studies have examined the molecular characteristics of the intracranial artery. ${ }^{8}$ However, recent technological advances have permitted amplification of the total RNA from vessel microsamples, facilitating transcriptomewide analysis of these specimens. In the present study, we sought to identify the gene expression profile specific to the intracranial artery of MMD. We collected microsamples of the MCA from patients with MMD and from control patients and performed transcriptome-wide analysis using the microarray technique.

\section{Methods}

This was a single-center, prospectively sampled, retrospective cohort study. The study protocol was carried out in agreement with the Declaration of Helsinki and was approved by the IRB of the Nagoya University Graduate School of Medicine. Written informed consent was obtained from all study participants or legal guardians. Figure $1 \mathrm{~A}$ provides a flow diagram of this study.

\section{Patient Characteristics}

A total of 20 Japanese patients who received surgical treatment at Nagoya University Hospital were included in this study. Detailed clinical information of the patients is shown in Supplemental Table 1. We obtained 11 microsamples of the MCA from patients with MMD and 9 microsamples of the MCA from control patients. In the MMD group, all patients received surgical revascularization including STA-MCA anastomosis. In the control group, 6 patients with an internal carotid artery aneurysm received aneurysm trapping or proximal ligation that required STA-MCA anastomoses as flow constructions, and 3 patients with medically intractable seizures received epileptic focus resection. The control patients were enrolled in a consecutive manner, and patients with MMD were selected to match the age, sex, and prevalence of underlying diseases regarding arteriosclerosis (hypertension, hyperlipidemia, and diabetes mellitus) of the control patients. The diagnosis of MMD was made according to the guidelines proposed by the Ministry of Health and Welfare of Japan. ${ }^{9}$ Briefly, the diagnostic criteria were as follows: 1) stenosis or occlusion around the terminal portion of the internal carotid arteries, 2) moyamoya vessels at the brain base, and 3) exclusion of diseases with similar angiographic characteristics (e.g., arteriosclerosis, autoimmune disease, meningitis, brain neoplasm, Down syndrome, neurofibromatosis type 1 , head trauma, or irradiation of the head). ${ }^{9,10}$ Figure $1 \mathrm{~B}$ shows the angiogram obtained in one such patient with MMD.

\section{Collection of Vessel Samples}

In each of the patients with MMD or with an internal carotid artery aneurysm, we collected a microsample of the MCA and a small sample of the STA during the 
STA-MCA anastomosis. STA-MCA anastomosis was performed in an end-to-side fashion with 10-0 or 11-0 nylon threads. ${ }^{11-13}$ The vessel wall of the MCA was excised following the reported procedure. ${ }^{6}$ Tissue samples were washed with normal saline containing $10 \mathrm{U} / \mathrm{mL}$ heparin, collected into RNAlater (Qiagen), and stored at $-80^{\circ} \mathrm{C}$. Figure $1 \mathrm{C}$ shows an intraoperative image of MCA arteriotomy in a patient with MMD. All STA-MCA anastomoses were performed successfully.

In the patients with intractable seizures who received epileptic focus resection, the cortical arteries were dissected from the resected tissue, washed with normal saline containing heparin, collected into RNAlater, and stored at $-80^{\circ} \mathrm{C}$.

\section{Detection of RNF213 c.14576G>A Variant}

Using the QIAamp UCP DNA Micro Kit (Qiagen), we obtained genomic DNA from the MCA samples of the patients with medically intractable seizures, or the STA samples of the patients with MMD or an internal carotid artery aneurysm. Exon 61 of $R N F 213$ was amplified via polymerase chain reaction (PCR) using a QuantiFast SYBR PCR Kit (Qiagen) with the manufacturer's recommended cycling conditions. The primer sequences are described in Supplemental Table 2. ${ }^{3}$ After gel electrophoresis, the PCR product was extracted using a FASTGene Gel/PCR extraction kit (Nippon Genetics). Presence of the c. $14576 \mathrm{G}>\mathrm{A}$ variant of $R N F 213$ was determined by Sanger sequencing with the Big Dye Terminator version 3.1 Cycle Kit and an ABI 3500 Genetic Analyzer (Applied Biosystems).

\section{RNA Extraction and Microarray Experiments}

Total RNA was extracted from each MCA specimen with an RNeasy Micro Kit (Qiagen). The purified total RNA was amplified into complementary DNA (cDNA) using an Ovation PicoSL WTA System V2 kit (NuGEN Technologies) and labeled with the SureTag DNA labeling kit (Agilent Technologies); these procedures were performed according to the respective manufacturer's instructions. Cy3-labeled amplified cDNA samples $(2 \mu \mathrm{g})$, prepared without the fragmentation reaction, were used for hybridization $\left(24\right.$ hours at $\left.65^{\circ} \mathrm{C}\right)$ to an oligonucleotide spotted microarray slide (Human Whole Genome, SurePrint G3 Human GE 8×60 K V2 microarray, Agilent Technologies, Inc.). Subsequent washing and drying were performed according to the Agilent hybridization protocol, and the slide was then scanned with an Agilent Model G2505A DNA microarray scanner.

\section{Microarray Data Analysis}

Using Feature Extraction Software version 11.0.1.1 (Agilent Technologies, Inc.), signal intensity was quantified from the scanned images with background correction. These signal intensities were normalized by shifting to 75th percentiles, as recommended in the Agilent One-Color Microarray-Based Gene Expression Analysis protocol. To reveal the approximate transcriptome profile distribution among the participants, principal component analysis was performed with all of the normalized values.
TABLE 1. Demographics of participants

\begin{tabular}{lccc}
\hline & MMD Group & Control Group & $p$ Value \\
\hline No. of patients & 11 & 9 & \\
\hline Age, yrs & & & 0.068 \\
\hline \multicolumn{1}{c}{ Median (IQR) } & $49(47.5-52.0)$ & $65(56.0-71.0)$ & \\
\hline \multicolumn{1}{c}{ Minimum-maximum } & $43-52$ & $14-79$ & \\
\hline Female sex & $10(90.9)$ & $6(66.7)$ & 0.29 \\
\hline Hypertension & $3(27.3)$ & $3(33.3)$ & 1.0 \\
\hline Hyperlipidemia & $4(36.4)$ & $3(33.3)$ & 1.0 \\
\hline Diabetes mellitus & $1(9.1)$ & $1(11.1)$ & 1.0 \\
\hline $\begin{array}{l}\text { c.14576G>A variant (G/A) } \\
\text { in RNF213 }\end{array}$ & $8(72.7)$ & $1(11.1)$ & 0.0098 \\
\hline
\end{tabular}

Values represent the number of patients (\%) unless indicated otherwise. Statistical analyses were performed using the Mann-Whitney U-test for age and the Fisher's exact test for categorical variables. The frequency of the c.14576G $>A$ variant in RNF213 was significantly higher in the MMD group. Other factors did not show significant differences.

To detect the differentially expressed genes in the MCA, we compared the microarray data between the MMD and control groups. Statistical analysis was performed with 18,596 genes whose RefSeq accessions have NM_ prefixes. The criteria to identify reliable differential gene expression were as follows: $:^{14,15} 1$ ) the Mann-Whitney U-test yielded a p value $<0.001$, and 2 ) the fold change exceeded 2. To understand the functional implication of differentially expressed genes, a gene ontology analysis was performed for biological process, molecular function, and cellular component using Metascape (https://metascape. org/). ${ }^{16,17}$ Gene interaction networks were analyzed using the GeneMANIA Cytoscape plugin of Cytoscape software version 3.8.2 (National Institute of General Medical Sciences, http://www.cytoscape.org/). ${ }^{18,19}$ Because the frequency of the c.14576G $>\mathrm{A}$ variant in RNF213 differed significantly between the MMD and control groups, we conducted gene interaction network analysis for 2 gene sets: 1) the differentially expressed genes, and 2) the differentially expressed genes and RNF213.

In addition, to investigate whether any specific gene sets were relevant to the gene expression of the MCA in the patients with MMD, gene set enrichment analysis (GSEA) was performed using the GSEA software program version 4.0.3 (Broad Institute, http://software.broadinstitute.org/ gsea/index.jsp). All the normalized values obtained from the microarray experiments were run against the hallmark gene sets, BIOCARTA subset of canonical pathways gene sets, and KEGG subset of canonical pathways gene sets (Molecular Signatures Database version 7.1, Broad Institute). The cutoff criterion was defined as a false discovery rate $<0.25$ according to the manufacturer's instructions. ${ }^{16}$ The microarray data are available in the Gene Expression Omnibus database (https://www.ncbi.nlm.nih.gov/geo/) under accession number GSE157628.

\section{Validation of Microarray Analysis With Quantitative PCR}

For quantitative PCR (qPCR) validation, we selected 5 genes according to the following criteria: 1) the genes 


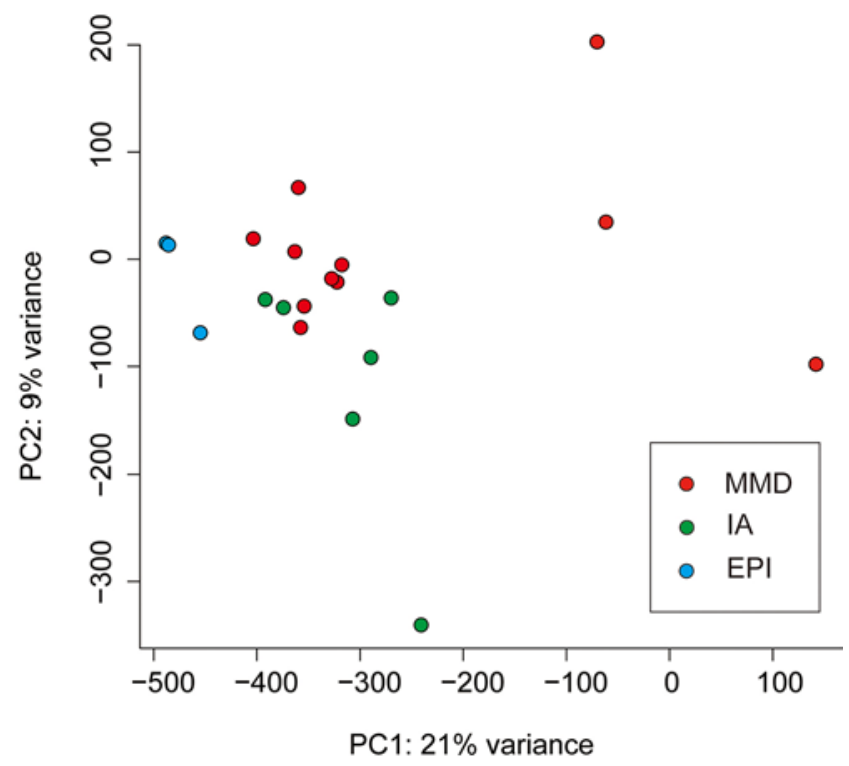

FIG. 2. Principal component (PC) analysis biplot for PC1 and PC2, performed using all of the normalized values obtained from the microarray experiments. Red dots represent patients with MMD, green dots represent patients with an internal carotid artery aneurysm $(\mathrm{IA})$, and light blue dots represent participants with medically intractable seizures (EPI).

showed significant differential expression in microarray analysis; 2) the genes exhibited near-average raw signal intensities in microarray analysis compared with the signal intensities of glyceraldehyde 3-phosphate dehydrogenase $(G A P D H)$, thereby facilitating reliable qPCR analysis; and $3)$ gene functions were of biological interest, such that the loci were expected to contribute to the pathophysiology of MMD. To measure gene expression levels, qPCR was conducted on an AriaMx Real-Time PCR System (Agilent Technologies, Inc.) using a QuantiFast SYBR PCR Kit (Qiagen) with the manufacturer's recommended cycling conditions. All samples were run in triplicate. Melting curve analysis and gel electrophoresis were performed to confirm the specific amplification of the qPCR product.

Gene expression was determined by the comparative $\mathrm{Ct}$ method; expression was normalized to that of GAPDH. The primer sequences are listed in Supplemental Table 2.

\section{Statistical Analysis}

Demographic variables were compared between groups using the Mann-Whitney U-test or the Fisher's exact test. Significance was set at $\mathrm{p}<0.05$. For microarray analysis, we applied the Mann-Whitney U-test for comparing gene expression between groups. The genes with a p value $<$ 0.001 and a fold change $>2$ were considered differentially expressed..$^{14,15}$ In GSEA, the cutoff criterion was set at a false discovery rate $<0.25 .^{20}$ For qPCR analysis, we also used the Mann-Whitney U-test and considered the genes with a $\mathrm{p}$ value $<0.05$ as differentially expressed. Statistical analyses were performed in $\mathrm{R}$ version 4.0.2 (https:// www.r-project.org/).
TABLE 2. Differentially upregulated genes in the MCA of patients with MMD compared with those in the MCA of control patients

\begin{tabular}{|c|c|c|c|}
\hline Gene & Accession & Fold Change & $p$ Value \\
\hline CBLN2 & NM_182511 & 6.3 & 2.3E-04 \\
\hline MATN1 & NM_002379 & 5.3 & 3.6E-04 \\
\hline MIA & NM_006533 & 5 & 8.0E-04 \\
\hline SP140 & NM_001005176 & 4.3 & 8.0E-04 \\
\hline NLGN4Y & NM_001164238 & 4.2 & $4.8 \mathrm{E}-05$ \\
\hline KIT & NM_000222 & 4 & 8.0E-04 \\
\hline FAM71A & NM_153606 & 4 & 8.0E-04 \\
\hline TTN & NM_133379 & 3.8 & 3.6E-04 \\
\hline ARHGEF39 & NM_032818 & 3.8 & 8.0E-04 \\
\hline OR4D11 & NM_001004706 & 3.5 & 1.4E-04 \\
\hline NECAB2 & NM_019065 & 3.4 & 4.8E-05 \\
\hline APCS & NM_001639 & 3.4 & 2.3E-04 \\
\hline SLC39A12 & NM_152725 & 3.3 & 1.4E-04 \\
\hline SLC37A2 & NM_001145290 & 3.3 & 1.4E-04 \\
\hline PTPRO & NM_030667 & 3.3 & 1.4E-04 \\
\hline LAMP3 & NM_014398 & 3.3 & 5.4E-04 \\
\hline FAT2 & NM_001447 & 3.3 & 5.4E-04 \\
\hline CDY2A & NM_004825 & 3.3 & 8.0E-04 \\
\hline ILIRAP & NM_001167931 & 3.2 & 5.4E-04 \\
\hline LIPM & NM_001128215 & 3.1 & $3.6 \mathrm{E}-04$ \\
\hline NOTUM & NM_178493 & 3.1 & 8.0E-04 \\
\hline POLR2F & NM_001301130 & 3 & 1.4E-04 \\
\hline$G A B R Q$ & NM_018558 & 3 & 5.4E-04 \\
\hline MCM5 & NM_006739 & 3 & 5.4E-04 \\
\hline OR7A5 & NM_017506 & 3 & 8.0E-04 \\
\hline CDHR3 & NM_152750 & 3 & 8.0E-04 \\
\hline SIGLEC8 & NM_014442 & 3 & 8.0E-04 \\
\hline OR2F1 & NM_012369 & 2.9 & 1.4E-04 \\
\hline IL10 & NM_000572 & 2.9 & 2.3E-04 \\
\hline MGAT5B & NM_144677 & 2.9 & 3.6E-04 \\
\hline ABCC6 & NM_001171 & 2.9 & 3.6E-04 \\
\hline FBXO24 & NM_033506 & 2.9 & 3.6E-04 \\
\hline B3GALT5 & NM_033171 & 2.9 & $5.4 \mathrm{E}-04$ \\
\hline OR13J1 & NM_001004487 & 2.9 & 5.4E-04 \\
\hline OPN1MW & NM_000513 & 2.8 & 1.4E-04 \\
\hline KNDC1 & NM_152643 & 2.8 & $3.6 \mathrm{E}-04$ \\
\hline RLBP1 & NM_000326 & 2.7 & $4.8 \mathrm{E}-05$ \\
\hline LOC101929983 & NM_001300891 & 2.7 & 3.6E-04 \\
\hline SLC19A3 & NM_025243 & 2.7 & 8.0E-04 \\
\hline MZB1 & NM_016459 & 2.6 & 3.6E-04 \\
\hline CERS3 & NM_178842 & 2.6 & 8.0E-04 \\
\hline MRGPRE & NM_001039165 & 2.5 & 8.3E-05 \\
\hline ACOXL & NM_001142807 & 2.5 & 1.4E-04 \\
\hline HLA-C & NM_001243042 & 2.5 & 3.6E-04 \\
\hline ZNF714 & NM_182515 & 2.5 & 8.0E-04 \\
\hline C3AR1 & NM_004054 & 2.4 & $4.8 \mathrm{E}-05$ \\
\hline PYDC2 & NM_001083308 & 2.4 & $3.6 \mathrm{E}-04$ \\
\hline CGN & NM_020770 & 2.4 & 8.0E-04 \\
\hline
\end{tabular}

CONTINUED ON PAGE 5 » 
» CONTINUED FROM PAGE 4

TABLE 2. Differentially upregulated genes in the MCA of patients with MMD compared with those in the MCA of control patients

\begin{tabular}{llcc}
\hline \multicolumn{1}{c}{ Gene } & \multicolumn{1}{c}{ Accession } & Fold Change & p Value \\
\hline OSBP2 & NM_030758 & 2.4 & $8.0 \mathrm{E}-04$ \\
\hline RASAL3 & NM_022904 & 2.3 & $8.0 \mathrm{E}-04$ \\
\hline CCDC37 & NM_182628 & 2.2 & $4.8 \mathrm{E}-05$ \\
\hline LOC102724279 & NM_001302493 & 2.2 & $3.6 \mathrm{E}-04$ \\
\hline RIIAD1 & NM_001144956 & 2.2 & $5.4 \mathrm{E}-04$ \\
\hline ASTL & NM_001002036 & 2.2 & $5.4 \mathrm{E}-04$ \\
\hline RET & NM_020630 & 2.2 & $8.0 \mathrm{E}-04$ \\
\hline STRA6 & NM_001142620 & 2.1 & $5.4 \mathrm{E}-04$ \\
\hline CREG2 & NM_153836 & 2.1 & $5.4 \mathrm{E}-04$ \\
\hline FAM178B & NM_016490 & 2.1 & $8.0 \mathrm{E}-04$ \\
\hline MS4A5 & NM_023945 & 2.1 & $8.0 \mathrm{E}-04$ \\
\hline TLDC2 & NM_080628 & 2.1 & $8.0 \mathrm{E}-04$ \\
\hline HIPK2 & NM_022740 & 2.1 & $8.0 \mathrm{E}-04$ \\
\hline DOCK9 & NM_001130050 & 2.1 & $8.0 \mathrm{E}-04$ \\
\hline
\end{tabular}

Genes that demonstrated $a>2$ fold change and showed a $p$ value $<0.001$ are listed. Statistical analyses were performed using the Mann-Whitney U-test.

\section{Results}

\section{Demographics of Participants}

Patient characteristics are summarized in Table 1. The profile of the 11 patients with MMD was compared with that of the 9 control patients. There were no significant differences in age, sex, and underlying disease regarding arteriosclerosis. The c. $14576 \mathrm{G}>\mathrm{A}$ variant in RNF213 detected in the participants was consistently heterozygous, and the rate of the $.14576 \mathrm{G}>\mathrm{A}$ variant in $R N F 213$ was significantly higher in the MMD group than in the control group $(p=0.010)$.

\section{Microarray Analysis}

Principal component analysis using the MCA transcriptomes of the participants showed relatively distinct clusters for the MMD and control groups (Fig. 2).

By comparing the MCA transcriptomes of patients with MMD with those of the control patients, we identified 62 upregulated and 26 downregulated differentially expressed genes (Tables 2 and 3). A volcano plot shows the differentially expressed genes visually, and a heatmap using these genes demonstrates a good clustering (Fig. 3). Gene ontology analysis identified several relatively associated terms $(\mathrm{p}<0.05)$ for these differentially expressed genes, including regulation of cell morphogenesis, protein tyrosine kinase activity, ubiquitin-protein transferase activity, interaction with host, myeloid cell differentiation, and leukocyte proliferation (Supplemental Table 3). In addition, the results of gene interaction network analysis are shown in Supplemental Fig. 1.

GSEA showed that genes involved with the cytokine pathway, dendritic cell (DC) pathway, interleukin-12 (IL12) pathway, and antigen processing and presentation exhibited significant positive correlations with gene expres-
TABLE 3. Differentially downregulated genes in the MCA of patients with MMD compared with those in the MCA of control patients

\begin{tabular}{|c|c|c|c|}
\hline Gene & Accession & Fold Change & $p$ Value \\
\hline ZNF880 & NM_001145434 & 12.8 & $2.3 \mathrm{E}-04$ \\
\hline SOCS7 & NM_014598 & 10.5 & $5.4 \mathrm{E}-04$ \\
\hline SPATA13 & NM_001286792 & 10.4 & $5.4 \mathrm{E}-04$ \\
\hline SGMS2 & NM_152621 & 7.7 & $2.3 \mathrm{E}-04$ \\
\hline GNPDA2 & NM_138335 & 7.6 & $1.4 \mathrm{E}-04$ \\
\hline CAMK2D & NM_001221 & 7.4 & $3.6 \mathrm{E}-04$ \\
\hline LYPLA1 & NM_006330 & 7.3 & 8.0E-04 \\
\hline SPRTN & NM_032018 & 7.2 & 8.0E-04 \\
\hline SLAIN1 & NM_001040153 & 6.9 & 8.0E-04 \\
\hline DUSP16 & NM_030640 & 6.4 & 8.0E-04 \\
\hline TUFT1 & NM_020127 & 5.8 & 8.0E-04 \\
\hline SH3BGRL2 & NM_031469 & 5.5 & $2.4 \mathrm{E}-05$ \\
\hline LRTOMT & NM_145309 & 5.5 & 8.0E-04 \\
\hline TSPAN2 & NM_005725 & 4.2 & $5.4 \mathrm{E}-04$ \\
\hline FADS3 & NM_021727 & 4 & $8.3 \mathrm{E}-05$ \\
\hline UBE2W & NM_001001481 & 4 & 8.0E-04 \\
\hline TNFRSF12A & NM_016639 & 3.9 & $5.4 \mathrm{E}-04$ \\
\hline$R H O Q$ & NM_012249 & 3.7 & 8.0E-04 \\
\hline DNTTIP2 & NM_014597 & 3.6 & 8.0E-04 \\
\hline CNOT4 & NM_001008225 & 3.4 & $3.6 \mathrm{E}-04$ \\
\hline CPED1 & NM_024913 & 3.1 & $8.0 \mathrm{E}-04$ \\
\hline TRIM44 & NM_017583 & 2.8 & $5.4 \mathrm{E}-04$ \\
\hline ODC1 & NM_002539 & 2.5 & $3.6 \mathrm{E}-04$ \\
\hline SEPT7 & NM_001788 & 2.4 & $1.2 \mathrm{E}-05$ \\
\hline HUWE1 & NM_031407 & 2.3 & $5.4 \mathrm{E}-04$ \\
\hline TTC3 & NM_003316 & 2.1 & 8.0E-04 \\
\hline
\end{tabular}

Genes that demonstrated $a>2$ fold change and showed a $p$ value $<0.001$ are listed. Statistical analyses were performed using the Mann-Whitney U-test.

sion in the MCA of the patients with MMD. In contrast, GSEA showed that genes involved in oxidative phosphorylation and DNA repair demonstrated a significant negative correlation with gene expression in the MCA of the patients with MMD (Fig. 4).

\section{Quantitative PCR Validation}

Following the criteria described in Methods, tetraspanin 2 (TSPAN2); ras homolog family member Q $(R H O Q)$; HECT, UBA, and WWE domain containing E3 ubiquitin-protein ligase 1 (HUWE1); homeodomaininteracting protein kinase 2 (HIPK2); and RAS protein activator-like 3 (RASAL3) were chosen for validation. The microarray and qPCR results of these transcripts are summarized in Fig. 5. The gene expression of GAPDH, the internal reference gene, was stable and consistent across the participants (Supplemental Fig. 2).

Comparing the expression of these transcripts between the MMD and control groups, qPCR analysis demonstrated similar distribution in gene expression as that observed in the microarray analysis. In addition, the decreased gene 

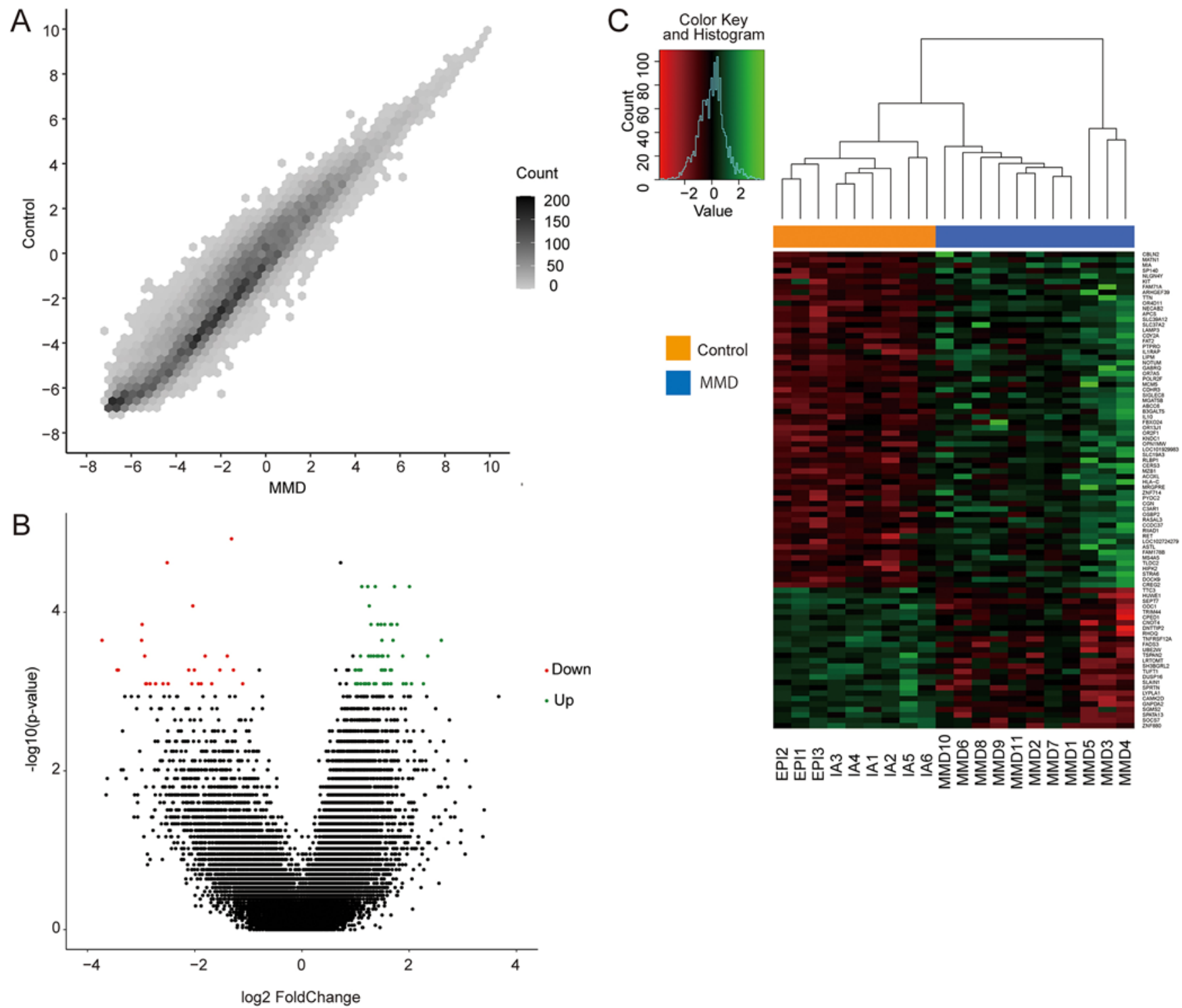

FIG. 3. Comparison of gene expression in microarray analyses between the MMD and control groups. A: Hexbin plot to assess the variation. The average normalized values of the individual gene in each group were used for construction. B: Volcano plot to visualize the differentially expressed genes. Green dots represent significantly (Mann-Whitney U-test, $p<0.001$ and fold change $>$ 2 ) upregulated genes $(n=62)$, and red dots represent significantly downregulated genes $(n=26)$. Black dots represent genes not differentially expressed. C: Heatmap comparing 88 differentially expressed genes across 20 samples (11 MMD and 9 control MCA specimens). Hierarchical clustering based on Euclidean distances is shown at the top. Row $Z$ scores of the normalized values were used for construction.

expression of TSPAN2 and RHOQ in the MCA of the patient with MMD was confirmed (TSPAN2, $\mathrm{p}=0.0074$; $R H O Q, \mathrm{p}=0.0097)$.

\section{Discussion}

Using microarray analysis, we showed differential gene expression in the MCA of the patients with MMD compared with that in the control patients. Based on GSEA, genes involved in antigen processing and presentation, and the DC pathway, cytokine pathway, and IL-12 pathway showed positive correlations to expression in the MCA of patients with MMD, while genes involved in oxidative phosphorylation and DNA repair showed negative correlations. Microarray analysis was validated by qPCR.

This study revealed that pathways associated with immune response were upregulated in the intracranial artery of patients with MMD: antigen processing and presentation, DC pathway, cytokine pathway, and IL-12 pathway. DCs are a group of antigen-presenting cells with roles in the initiation and regulation of both innate and adaptive immune responses. ${ }^{21}$ IL-12 is primarily a proinflammatory and prostimulatory cytokine produced by inflammatory myeloid cells, and this molecule plays a key role 

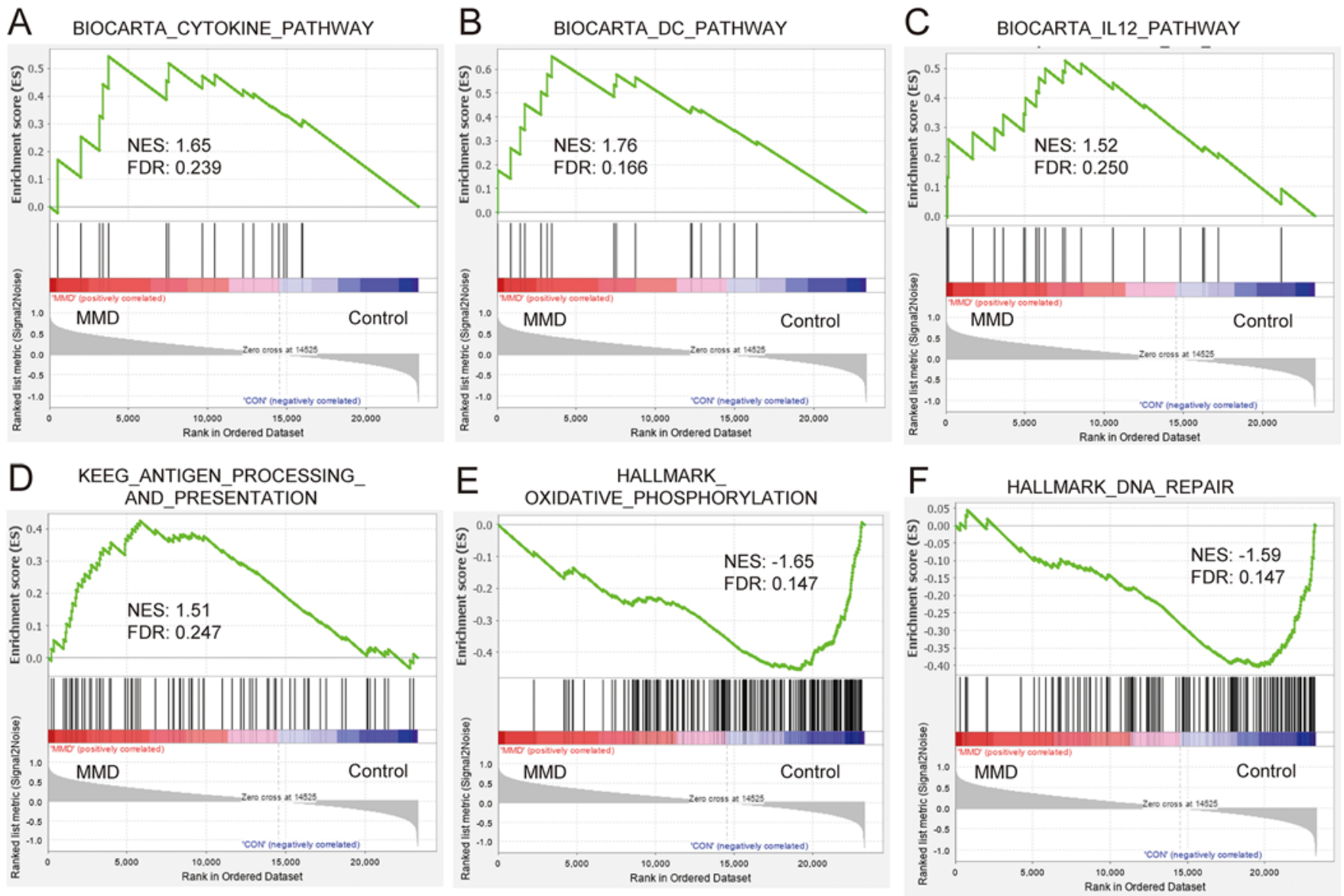

FIG. 4. GSEA enrichment score plots of the indicated gene sets for the MCA of patients with MMD and control patients. GSEA revealed that the gene sets of the cytokine pathway, DC pathway, IL-12 pathway, and antigen processing and presentation showed positive correlations with gene expression in the MCA of patients with MMD (A-D), and the gene sets of oxidative phosphorylation and DNA repair showed negative correlations $(E$ and $F)$. FDR = false discovery rate; NES = normalized enrichment score.

in the development of the $\mathrm{T}_{\mathrm{H}} 1$ subset of helper $\mathrm{T}$ cells. ${ }^{22}$ Our results are consistent with those of several previous reports. ${ }^{23,24}$ Specifically, in a microarray study using blood samples from patients with MMD, integrated analysis of long noncoding RNA-messenger RNA coexpression networks were linked to the inflammatory response, Toll-like signaling pathway, and cytokine-cytokine receptor interactions. ${ }^{23}$ In histopathological studies with specimens obtained at autopsy, Masuda et al. showed the colocalization of proliferating smooth muscle cells and inflammatory cells such as macrophages or $\mathrm{T}$ cells in the thickened intima of occlusive major intracranial arteries. ${ }^{24}$ However, the detailed mechanism of immune response in MMD is still unknown, and further studies are needed.

The downregulation of genes involved in oxidative phosphorylation in the MCA of patients with MMD is another important finding. The oxidative phosphorylation system is the final biochemical pathway in the production of adenosine triphosphate, and the system is embedded in the lipid bilayer of the mitochondrial inner membrane..$^{25}$ In in vitro experiments using endothelial colony-forming cells from patients with MMD, the mitochondria were shorter, displayed a circular morphology, and showed de- creased oxygen consumption rates; these are properties that indicate the functional abnormality of oxidative phosphorylation. In addition, angiogenic activity is impaired in patients with MMD. ${ }^{26}$ Our results and the prevalent differences in ethnicity or sex also support the possibility of mitochondrial association with the pathogenesis of MMD..$^{27,28}$

The expression of genes involved in DNA repair was also downregulated in the MCA of patients with MMD. Defective DNA repair can lead to vasodilator dysfunction or increased vascular stiffness, ${ }^{29}$ but the cause or effect of defective DNA repair in MMD has not been studied. However, the c.14576G $>\mathrm{A}$ variant in $R N F 213$ is expected to serve as a target for further research. RNF213 encodes a huge protein containing a really interesting new gene (RING) domain indicating the presence of E3 ubiquitin ligase activity. ${ }^{3}$ Considering that some E3 ubiquitin ligases are involved in the physiological activity of DNA repair, ${ }^{30}$ the ubiquitin ligase activity of RNF213 and the effect of mutations in this gene, which has been the subject of a small number of studies, ${ }^{31}$ might be deserving of investigation.

In qPCR analyses, TSPAN2 and RHOQ showed signifi- 

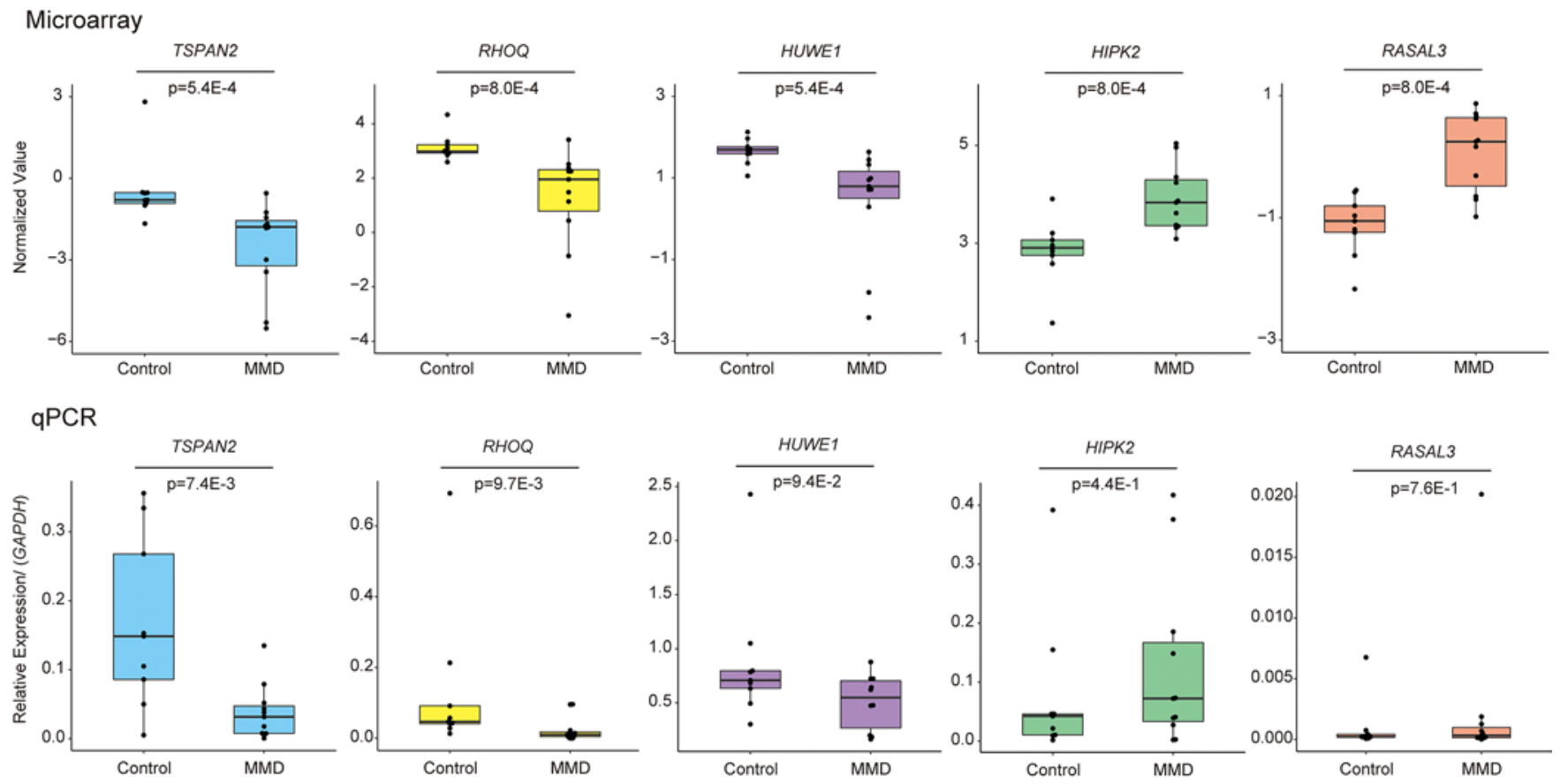

FIG. 5. Comparison of the gene expression level between the MMD and control groups. Upper: Box and beeswarm plots representing the expression level in microarray experiments. The normalized values were used for construction. Lower: Box and beeswarm plots representing the expression level measured by the qPCR analyses. The expression is normalized to GAPDH. TSPAN2 and RHOQ showed significant differential expression in the MCA of patients with MMD compared with those patients with control conditions. Statistical analyses were performed by the Mann-Whitney U-test.

cantly decreased expression in the MCA of patients with MMD. TSPAN2 is a 4-transmembrane-domain protein that is highly expressed in smooth muscle-enriched tissues; this protein has functions in suppressing vascular smooth muscle cell proliferation and migration. ${ }^{32}$ Decreased expression of TSPAN2 might be a cause of the smooth muscle proliferation observed in the intracranial artery of patients with MMD. ${ }^{24} R H O Q$ is a member of the small Rho GTPase family. Loss of $R H O Q$ expression has been shown to decrease the level of signaling by Notch, a protein that is important for ensuring the appropriate response of endothelial cells to proangiogenic stimuli; silencing of $R H O Q$ has been shown to result in abnormal blood vessel sprouting and formation, in both in vivo and in vitro experiments. ${ }^{33}$ Decreased expression of $R H O Q$ can alter the angiographical characteristics of MMD, such as the abnormal collateral vessels. ${ }^{1}$

This study has some limitations. The first issue is the selection of control samples. Because the molecular characteristics of the arterial vessels differ depending on their location, their profiles should be compared with those of arteries of the same location. ${ }^{34}$ The intracranial arteries of healthy patients may be the optimal control samples, but obtaining such samples is ethically problematic. Therefore, we included patients with intracranial artery aneurysms and medically intractable seizures, from whom we could obtain intracranial cortical arteries without lesions. Second, we should consider the differences between ethnicities in MMD. In the present study, all of the participants were Japanese, and most of the patients with
MMD harbored the c.14576G $>$ A variant in $R N F 213$. We have discussed the possible associations of mitochondrial function and the RNF213 variants with the molecular characteristics in the MCA of MMD. The mitochondrial haplogroup and the variant type or frequency of relevant alleles of RNF213 differ between Japanese and European patients, ${ }^{35,36}$ thus, it is unclear whether our findings can be generalized to European patients. Third, the possible effects on the gene expression by the cohort characteristics should be considered. It is unknown how differences among the participants (e.g., medicines taken, clinical phenotypes, and duration from the last symptoms until the surgeries) affect the gene expression in the MCA. In addition, although not statistically significant, there were relative differences in age and sex between the groups in our study. Fourth, RNA quality was not measured in this study. Our research employed MCA specimens that were tiny, and the amounts of total RNA obtained from these specimens were small, making it necessary to use all of the obtained total RNA for cDNA amplification. Overall, our findings are limited by the possible bias in control group selection; the difficulty in generalizing our results due to the limited ethnic variety of the included patients with MMD; and the need for further validation using protein analysis, in vitro investigation of mRNA function, and better cohort stratification.

\section{Conclusions}

This transcriptome-wide analysis showed upregulation 
of genes associated with immune response and downregulation of genes involved in oxidative phosphorylation and DNA repair within the intracranial arteries of patients with MMD. These findings may provide important clues for further studies to clarify the pathophysiology of MMD.

\section{Acknowledgments}

We thank Ms. Akiko Shimada for her technical help with the experiments. This work was funded by KAKENHI grants from the Japan Society for the Promotion of Science to Y. Araki (No. 7118K08967) and K. Yokoyama (No. 20K17961).

\section{References}

1. Kuroda S, Houkin K. Moyamoya disease: current concepts and future perspectives. Lancet Neurol. 2008;7(11):1056-1066.

2. Miyamoto S, Yoshimoto T, Hashimoto N, Okada Y, Tsuji I, Tominaga T, et al. Effects of extracranial-intracranial bypass for patients with hemorrhagic moyamoya disease: results of the Japan Adult Moyamoya Trial. Stroke. 2014;45(5):14151421.

3. Liu W, Morito D, Takashima S, Mineharu Y, Kobayashi H, Hitomi T, et al. Identification of RNF213 as a susceptibility gene for moyamoya disease and its possible role in vascular development. PLoS One. 2011;6(7):e22542.

4. Kamada F, Aoki Y, Narisawa A, Abe Y, Komatsuzaki S, Kikuchi A, et al. A genome-wide association study identifies RNF213 as the first Moyamoya disease gene. J Hum Genet. 2011;56(1):34-40.

5. Hosoda Y, Ikeda E, Hirose S. Histopathological studies on spontaneous occlusion of the circle of Willis (cerebrovascular moyamoya disease). Clin Neurol Neurosurg. 1997;99(suppl 2):S203-S208.

6. Takagi Y, Kikuta K, Sadamasa N, Nozaki K, Hashimoto N. Caspase-3-dependent apoptosis in middle cerebral arteries in patients with moyamoya disease. Neurosurgery. 2006;59(4): 894-901.

7. Takagi Y, Kikuta K, Nozaki K, Fujimoto M, Hayashi J, Imamura H, Hashimoto N. Expression of hypoxia-inducing factor-1 alpha and endoglin in intimal hyperplasia of the middle cerebral artery of patients with Moyamoya disease. Neurosurgery. 2007;60(2):338-345.

8. Okami N, Aihara Y, Akagawa H, Yamaguchi K, Kawashima A, Yamamoto T, Okada Y. Network-based gene expression analysis of vascular wall of juvenile Moyamoya disease. Childs Nerv Syst. 2015;31(3):399-404.

9. Research Committee on the Pathology and Treatment of Spontaneous Occlusion of the Circle of Willis. Guidelines for diagnosis and treatment of moyamoya disease (spontaneous occlusion of the circle of Willis). Neurol Med Chir (Tokyo). 2012;52(5):245-266.

10. Nishimoto A, Takeuchi S. Abnormal cerebrovascular network related to the internal carotid arteries. J Neurosurg. 1968;29(3):255-260.

11. Yasargil MG, Krayenbuhl HA, Jacobson JH II. Microneurosurgical arterial reconstruction. Surgery. 1970;67(1):221-233.

12. Donaghy RM. Neurologic surgery. Surg Gynecol Obstet. 1972;134(2):269-270.

13. Kuroda S, Houkin K, Ishikawa T, Nakayama N, Iwasaki Y. Novel bypass surgery for moyamoya disease using pericranial flap: its impacts on cerebral hemodynamics and long-term outcome. Neurosurgery. 2010;66(6):1093-1101.

14. Ballman KV. Genetics and genomics: gene expression microarrays. Circulation. 208;118(15):1593-1597.

15. Shi L, Jones WD, Jensen RV, Harris SC, Perkins RG, Goodsaid FM, et al. The balance of reproducibility, sensitivity, and specificity of lists of differentially expressed genes in microarray studies. BMC Bioinformatics. 2008;9(suppl 9):S10.
16. The Gene Ontology Consortium. The Gene Ontology Resource: 20 years and still GOing strong. Nucleic Acids Res. 2019;47(D1):D330-D338.

17. Zhou Y, Zhou B, Pache L, Chang M, Khodabakhshi AH, Tanaseichuk O, et al. Metascape provides a biologist-oriented resource for the analysis of systems-level datasets. Nat Commun. 2019;10(1):1523.

18. Montojo J, Zuberi K, Rodriguez H, Kazi F, Wright G, Donaldson SL, et al. GeneMANIA Cytoscape plugin: fast gene function predictions on the desktop. Bioinformatics. 2010; 26(22):2927-2928.

19. Shannon P, Markiel A, Ozier O, Baliga NS, Wang JT, Ramage D, et al. Cytoscape: a software environment for integrated models of biomolecular interaction networks. Genome Res. 2003;13(11):2498-2504.

20. Subramanian A, Tamayo P, Mootha VK, Mukherjee S, Ebert BL, Gillette MA, et al. Gene set enrichment analysis: a knowledge-based approach for interpreting genome-wide expression profiles. Proc Natl Acad Sci U S A. 2005;102(43): 15545-15550.

21. Neefjes J, Jongsma ML, Paul P, Bakke O. Towards a systems understanding of MHC class I and MHC class II antigen presentation. Nat Rev Immunol. 2011;11(12):823-836.

22. Vignali DA, Kuchroo VK. IL-12 family cytokines: immunological playmakers. Nat Immunol. 2012;13(8):722-728.

23. Wang W, Gao F, Zhao Z, Wang H, Zhang L, Zhang D, et al. Integrated analysis of LncRNA-mRNA co-expression profiles in patients with moyamoya disease. Sci Rep. 2017;7: 42421.

24. Masuda J, Ogata J, Yutani C. Smooth muscle cell proliferation and localization of macrophages and T cells in the occlusive intracranial major arteries in moyamoya disease. Stroke. 1993;24(12):1960-1967.

25. Smeitink J, van den Heuvel L, DiMauro S. The genetics and pathology of oxidative phosphorylation. Nat Rev Genet. 2001; 2(5):342-352.

26. Choi JW, Son SM, Mook-Jung I, Moon YJ, Lee JY, Wang $\mathrm{KC}$, et al. Mitochondrial abnormalities related to the dysfunction of circulating endothelial colony-forming cells in moyamoya disease. J Neurosurg. 2018;129(5):1151-1159.

27. Uchino K, Johnston SC, Becker KJ, Tirschwell DL. Moyamoya disease in Washington State and California. Neurology. 2005;65(6):956-958.

28. Kuriyama S, Kusaka Y, Fujimura M, Wakai K, Tamakoshi A, Hashimoto S, et al. Prevalence and clinicoepidemiological features of moyamoya disease in Japan: findings from a nationwide epidemiological survey. Stroke. 2008;39(1):42-47.

29. Durik M, Kavousi M, van der Pluijm I, Isaacs A, Cheng C, Verdonk K, et al. Nucleotide excision DNA repair is associated with age-related vascular dysfunction. Circulation. 2012; 126(4):468-478.

30. Deshaies RJ, Joazeiro CA. RING domain E3 ubiquitin ligases. Annu Rev Biochem. 2009;78(1):399-434.

31. Takeda M, Tezuka T, Kim M, Choi J, Oichi Y, Kobayashi $\mathrm{H}$, et al. Moyamoya disease patient mutations in the RING domain of RNF213 reduce its ubiquitin ligase activity and enhance NFKB activation and apoptosis in an AAA+ domain-dependent manner. Biochem Biophys Res Commun. 2020;525(3):668-674.

32. Zhao J, Wu W, Zhang W, Lu YW, Tou E, Ye J, et al. Selective expression of TSPAN2 in vascular smooth muscle is independently regulated by TGF- $\beta 1 /$ SMAD and myocardin/serum response factor. FASEB J. 2017;31(6):2576-2591.

33. Bridges E, Sheldon H, Kleibeuker E, Ramberger E, Zois C, Barnard A, et al. RHOQ is induced by DLL4 and regulates angiogenesis by determining the intracellular route of the Notch intracellular domain. Angiogenesis. 2020;23(3):493513.

34. Laarman MD, Kleinloog R, Bakker MK, Rinkel GJE, Bak- 
kers J, Ruigrok YM. Assessment of the most optimal control tissue for intracranial aneurysm gene expression studies. Stroke. 2019;50(10):2933-2936.

35. Ingman M, Kaessmann H, Pääbo S, Gyllensten U. Mitochondrial genome variation and the origin of modern humans. Nature. 2000;408(6813):708-713.

36. Guey S, Kraemer M, Hervé D, Ludwig T, Kossorotoff M, Bergametti F, et al. Rare RNF213 variants in the C-terminal region encompassing the RING-finger domain are associated with moyamoya angiopathy in Caucasians. Eur J Hum Genet. 2017;25(8):995-1003.

\section{Disclosures}

The authors report no conflict of interest concerning the materials or methods used in this study or the findings specified in this paper.

\section{Author Contributions}

Conception and design: Yokoyama, Maruwaka. Acquisition of data: Yokoyama, Kanamori, Karnan, Shimizu, S Ota, Uda, Araki, Okamoto, Maesawa. Analysis and interpretation of data: Yokoyama, Kanamori, A Ota, Karnan, Shimizu. Drafting the article: Kanamori. Critically revising the article: Yokoyama, A Ota,
Yoshikawa, Natsume. Reviewed submitted version of manuscript: Yokoyama, A Ota, Yoshikawa, Karnan, Maruwaka, Shimizu, S Ota, Uda, Araki, Okamoto, Maesawa, Wakabayashi, Natsume. Statistical analysis: Yokoyama, Kanamori, A Ota, Karnan. Administrative/technical/material support: A Ota, Yoshikawa, Karnan. Study supervision: Yoshikawa, Wakabayashi, Natsume.

\section{Supplemental Information}

Online-Only Content

Supplemental material is available online.

Supplemental Tables and Figures. https://thejns.org/doi/suppl/ 10.3171/2021.6.FOCUS20870.

\section{Correspondence}

Kinya Yokoyama: Nagoya University Graduate School of Medicine, Nagoya City, Aichi, Japan. k-yokoyama@med. nagoya-u.ac.jp. 